\title{
Estilos de liderazgo de los directivos universitarios en Colombia
}

\author{
Leadership styles of the university managers in Colombia
}

\author{
GONZALEZ-CAMPO, Carlos H. ${ }^{1}$ \\ MURILLO-VARGAS, Guillermo ${ }^{2}$ \\ GARCIA-SOLARTE, Monica ${ }^{3}$
}

\begin{abstract}
Resumen
El objetivo de este artículo fue caracterizar los estilos de liderazgo del directivo universitario en Colombia. En la investigación se realizó una encuesta a 272 directivos de universidades colombianas. Para su analisis se utilizaron métodos cuantitativos con estadísticos descriptivos. Los resultados muestran que el estilo de liderazgo evidenciado es el transformacional siendo las mujeres quienes más lo reflejan. En la discusión se contrastan los resultados con la bibliografía existente. Finalmente, son presentadas las conclusiones.

Palabras clave: estilos de liderazgo, universidades, directivos.

Abstract

The objective of this paper was to characterize the leadership styles of the university manager in Colombia. The research carried out a survey of 272 executives from Colombian universities, for their analysis, quantitative methods with descriptive statistics were used. The results show that the leadership style evidenced is transformational leadership and that it is women who most reflect it. In the discussion, the results are compared with the existing bibliography. Finally, the conclusions are presented.

key words: leadership styles, universities, managers.
\end{abstract}

\section{Introducción}

La gestión y la dirección de las organizaciones de educación superior constituye hoy en día un tema de interés, debido a los cambios sociales y políticos que han movilizado al sector universitario hacia enfoques administrativos estratégicos, basados en las lógicas del mercado y la competitividad (Deem, 2004; Mercer, 2009). Los directivos universitarios son, por lo general, profesores de diversas áreas que incursionan en los procesos de dirección y, por tanto, deben potencializar sus competencias directivas (Esquivias, 2014). Cuando se estudia el directivo universitario, además de incluir el ejercicio de la gestión universitaria, se estudia el perfil, el género, la trayectoria y los valores, que inciden en la manera de dirigir. Sin embargo, su estilo de dirección debe estar

\footnotetext{
${ }^{1}$ Profesor Tiempo Completo. Departamento de Administración y Organizaciones. Universidad del Valle. Email:carlosh.gonzalez@correounivalle.edu.co 2 Profesor Tiempo Completo. Departamento de Administración y Organizaciones. Universidad del Valle. Email:guillermo.murillo@correounivalle.edu.co

3 Profesora Tiempo Completo. Departamento de Administración y Organizaciones. Universidad del Valle. Email:monica.garcia@correounivalle.edu.co
} 
enmarcado en las definiciones estratégicas de la institución, que en muchos casos está determinada en la misión, visión, valores, objetivos y estrategias (Lopera, 2004).

La gestión de los directivos y su estilo de liderazgo influye en el éxito o fracaso de la organización universitaria y de estos resultados y trayectoria se construye el perfil del mismo en el sector académico. Por lo tanto, su gestión y liderazgo estarán determinados por las competencias gerenciales, organizacionales y personales que posea el directivo, lo cual condiciona sus comportamientos, conductas y la manera en que asume su postura frente a las exigencias y dinámicas del entorno (Barbosa et al., 2017).

En Colombia, el desarrollo de la universidades se caracteriza por ser públicas o privadas, poseer distintas estructuras organizativas, distintas políticas de gestión y distintas afiliaciones religiosas (Soto, 2005; Beltrán, 2013). En Colombia existen 302 instituciones de educación superior, que incluyen a las universidades, otras instituciones universitarias, las instituciones tecnológicas y las instituciones técnicas profesionales, todas ellas conocidas como IES. Desde el punto de vista de los órganos máximos de decisión, en la mayoría de los casos es un consejo superior y el responsable de la IES, es un rector, encargado de la dirección académica y administrativa. Para la gestión, cada IES cuenta con una estructura organizacional, un conjunto de procesos y un plan estratégico para la ejecución de las actividades en un periodo de tiempo, desde donde se evalúa la gestión de los directivos.

Aunque son escasos los estudios en la gestión universitaria, algunos se han concentrado en identificar el modo en que es llevada a cabo (Brunner, 2011); Restrepo, Trujillo, y Guzmán, (2012), mientras que otros han intentado caracterizar a los sujetos que realizan este tipo de prácticas (Mercer, 2009); Winter, (2009); Castro y Tomàs, (2011). Una de las características de gestión que aportan a los procesos de transformación es el liderazgo, que es la capacidad para gestionar la fuerza de trabajo comprometida con la excelencia universitaria y la calidad institucional (Gómez,2020). Uno de los estilos de liderazgo estudiados es el transformacional, que se espera sea un liderazgo más propio de contextos inestables y de incertidumbre que un liderazgo transaccional, que es más propio de contextos estables y con mayor posibilidad de predicción (Contreras y Barbosa, 2013).

\subsection{Tipos de liderazgo de directivos universitarios}

Las instituciones de educación superior han tenido cambios importantes en los últimos años a nivel mundial, entre otros determinados por el uso de las TIC, la masificación y la globalización (Altbach, 2015). En este contexto, según Khalifa y Ayoubi (2015), los líderes o directivos universitarios se enfrentan a un mayor grado de incertidumbre y compiten por recursos. Hoy en día, las universidades deben asegurar el ofrecimiento del servicio educativo cumpliendo con estándares de calidad (Rodríguez-Ponce, 2009). Estos retos a los que se enfrentan las IES, requieren líderes que asuman la responsabilidad de estos cambios (Black, 2015), buscando mayores niveles de eficiencia y un mejor desempeño.

En este sentido, el líder tiene la responsabilidad de la transformación de las instituciones de educación superior; se requiere un liderazgo transformacional para cambiar la gestión jerárquica y burocrática. Como lo plantea Gómez (2020), para asegurar los cambios en la educación universitaria en el contexto actual, es necesario que estas instituciones modifiquen la manera de conducirse; esto significa un liderazgo que permita transformar la concepción que se tiene acerca de la realidad, a los fines de poder alinearse con las tendencias globales que operan en este importante sector. De acuerdo con González et al. (2013), en el contexto universitario esto implicaría que las universidades salieran de formas tradicionales de conducirse, acogiendo nuevas estrategias para inspirar a las personas. Los intereses de las organizaciones y sus miembros necesitan integrarse; esta es una tarea del liderazgo transformacional. El liderazgo transformacional es inspirador, estimula intelectualmente y considera individualmente a los trabajadores. Mendoza, et al.,(2007).

Para Hirtz, Murray y Riordan (2007), los resultados de su investigación en universidades en Estados Unidos muestran una relación positiva entre el estilo de liderazgo transformacional y el éxito en la gestión de la calidad 
de las instituciones, razón por la cual, en este proceso de transformación, el liderazgo del directivo universitario es un factor determinante del futuro de la institución.

\section{Liderazgo transformacional}

El liderazgo transformacional es un proceso que se da en la relación líder-seguidores, donde el líder motiva a los seguidores a hacer más de lo que se esperaba que hagan y los hace conscientes de la importancia de su puesto de trabajo para el cumplimiento de los objetivos organizacionales, buscando su transformación y la de la organización (Avolio y Atwater, 1996). Se divide en cuatro factores, como son: la «influencia idealizada», donde el líder se caracteriza por ser carismático y los seguidores se identifican con él y desean imitarlo; «motivación inspiracional», donde el líder inspira y motiva a los seguidores a través de desafíos alcanzables, dándoles significado a las metas tanto en lo personal como en lo organizacional; «estimulación individual», donde el líder impulsa a los seguidores a mejorar cada vez más sus capacidades y habilidades; y "consideración individualizada», donde el líder les brinda apoyo, guía y formación de manera individual (Avolio y Bass, 1995; Cuadrado, 2003).

\section{Liderazgo transaccional}

El liderazgo transaccional ha sido definido como la relación líder-seguidores, basada en una serie de intercambios o ganancias/sanción. Se divide en dos factores, como son: "recompensa contingente», donde el líder define y asigna los roles y las responsabilidades que se deben desarrollar para el logro de la meta y acuerda con los seguidores las tareas para obtener la recompensa o evitar la sanción que se estipule en un contrato, y «dirección por excepción", donde los seguidores esperan que el líder los habilite para lograr un resultado favorable y sienten que el intercambio es transparente y justo si las recompensas son distribuidas equitativamente (Avolio y Bass, 1995; Cuadrado, 2003). A diferencia del transformacional, el líder transaccional practica el reforzamiento contingente de los seguidores.

\section{Liderazgo Laissez-Faire}

El liderazgo Laissez-Faire es un liderazgo ausente; evita influenciar a sus subordinados, elude sus responsabilidades de supervisión y no confía en su habilidad para dirigir. Deja mucha responsabilidad sobre los empleados, no pone metas claras y no ayuda a su grupo a tomar decisiones, evitándolas (Avolio y Bass, 1995; Cuadrado, 2003).

\section{Liderazgo según el género}

En concordancia con investigaciones previas, no es posible determinar que los hombres tienen un estilo de liderazgo masculino mientras que las mujeres un liderazgo femenino. Para Oplatka (2006), cualquier estilo puede ser asumido por ambos. Para Moncayo-Orjuela y Zuluaga-Goyeneche (2015), es complejo afirmar que en las IES existe un liderazgo de hombres y otro de mujeres, asociado a estereotipos masculinos (competitividad, agresividad, determinación y autoritarismo) y femeninos (pasividad, emotividad y sensibilidad).

\section{Liderazgo universitario}

En el contexto universitario, también se han desarrollado propuestas para un liderazgo educativo. Para MoncayoOrjuela y Zuluaga--Goyeneche (2015), los estilos de liderazgo han pasado por tres momentos: los estudios centrados en la personalidad de líder, en donde se le atribuye una figura épica y se caracteriza por un hecho heroico; los estudios del liderazgo orientados a la postura, que vincula al liderazgo con los seguidores a través de la percepción de estos; y, finalmente, los estudios basados en el contexto, los cuales sugieren que el carisma de un líder está versado por su contexto y este puede cambiar a partir de diversos componentes. 
Algunos autores han realizado estudios a nivel de sus países.En el Reino Unido (Middlehurst, 2004), demuestra que se debe realizar un cambio de enfoque de la estructura y funciones de las personas y los procesos en la tarea de liderar el cambio en las universidades. Seale y Cross (2015), han estudiado a los decanos de las universidades en Sudáfrica, analizando el liderazgo para entender las realidades del contexto desde las capacidades individuales para mejorar el desempeño individual y organizacional.

En Latinoamérica, Ferrer, Colmenares y Clemenza (2010), en Venezuela, han realizado análisis de liderazgo ético como soporte de gestión estratégica en universidades venezolanas. En Colombia, Pérez, Jiménez y Romo (2017) han caracterizado el liderazgo transformacional de los directivos en una universidad del departamento de Antioquia, demostrando la pertinencia de este tipo de liderazgo.

La investigación sobre el liderazgo en los directivos universitarios permite dar cuenta de aquello que no se ve a simple vista, de lo que está oculto en la gestión y que solo se hace evidente a través de la indagación rigurosa y metodológicamente estructurada. En especial, en un campo de acción como la gestión universitaria, donde los diferentes actores en condiciones normales provienen de campos diferentes a la gestión administrativa y académica. Por otra parte, se hace absolutamente necesario dotar aquellos que están orientados a la gestión de organizaciones del conocimiento como son las universidades de los conceptos y características de aquello que deben considerar en las diferentes circunstancias de la gestión académica.

De ahí que el objetivo de este artículo es describir el estilo de liderazgo de los directivos universitarios en Colombia y analizar si hay diferencias de acuerdo al tipo de institución que dirigen. Este trabajo contribuye, en primer lugar, a los estudios empíricos de instituciones de educación superior, principalmente en Colombia, en donde la literatura sobre el estilo de liderazgo en la dirección universitaria es escasa y este estudio permite identificar cual es el estilo de liderazgo de los directivos. En segundo lugar, los estudios de las prácticas administrativas en las universidades se describen según el tipo de institución y el género del directivo, lo que permite aportar a las prácticas administrativas de acuerdo al contexto en el que se desarrollan.

\section{Metodología}

Para el cumplimiento del objetivo principal, este artículo realizó un estudio empírico con metodología cuantitativa, donde se utilizó como técnica de recolección el cuestionario, el cual se aplicó por medio de una encuesta dentro de la investigación, a una muestra aleatoria no probabilística de 272 directivos universitarios de diferentes universidades colombianas. La variable liderazgo se discrimina entre el liderazgo transformacional, el Laissez-Faire y el liderazgo transaccional desde lo propuesto por Avolio y Bass (1995). En este sentido, la investigacion es exploratoria, donde en el analisis de los datos de la encuesta realizada para este articulo, se utilizó el enfoque cuantitativo para realizar una indagacion de la percepcion de los directivos, y luego el analisis de la literatura revisada, para confirmar y contrastar algunos de los resultados previos.

\subsection{Fase cuantitativa}

Para la recolección de los datos cuantitativos se utilizó un cuestionario, el cual se propuso con preguntas cerradas, que se incluye en el Anexo 1.

\section{Muestra}

Para recoger los datos para este articulo, se realizó un muestreo por conveniencia en donde se encuestaron un total de 272 directivos universitarios de 74 universidades del país.

El instrumento se aplicó vía telefónica y se envió por correo electrónico durante los meses de agosto de 2017 y junio de 2018, tiempo en el que se obtuvieron 272 respuestas, lo que corresponde a una tasa de respuesta del 24\% aproximadamente del total de la población 1.134 directivos. 
La mayor parte de las universidades encuestadas se encuentran en Bogotá siendo el 59,9 \% privadas y el 39,7 \% públicas. Con relación al número de estudiantes, en promedio, las universidades cuentan con 19.378 estudiantes y el 67,3 \% cuenta con acreditación de Alta Calidad. Por otra parte, el $58.8 \%$ de los directivos eran hombres, mientras que el $41,2 \%$ eran mujeres, la mayoría de estos se encuentran entre los 47 y 58 años, con una media de 51 años. Con relación al nivel educativo, el 51,5\% son Magister, el 38,2 \% Doctores, el 7,7 \% especialistas, el $1,8 \%$ profesionales y tecnólogos el $0.7 \%$.

\section{Escalas}

Para determinar los estilos de liderazgo, en el cuestionario que soporta las encuestas realizadas para este articulo, se utilizó el "Multifactor Leadership Questionnaire" desarrollado por Bass y Avolio (1992), el cual contiene un total de 21 ítems que permite medir 7 factores: influencia idealizada, motivación inspiracional, estimulación intelectual, consideración individualizada, premios contingentes, excepción por la dirección y liderazgo Laissez-Faire. Estos factores a su vez, permiten identificar tres estilos de liderazgo: Transformacional, Transaccional y Laissez-Faire. Para este bloque se utilizó una escala tipo Likert de 1 a 5 (1= Nunca; 2= De vez en cuando; $3=$ Algunas veces; $4=$ Bastante a menudo; 5= Frecuentemente, sino es siempre).

\section{Análisis componente cuantitativo}

Una vez aplicada la encuesta, se determinan los estadísticos descriptivos, para evaluar la fiabilidad de los índices de liderazgo a través de las subvariables se realiza un análisis de fiabilidad con alfa de Cronbach. Para los 3 tipos de liderazgo se obtuvieron resultados favorables con el alfa de Cronbach: Liderazgo Transformacional $(0,778)$, Liderazgo Transaccional $(0,653)$ y Liderazgo Laissez-faire $(0,711)$. Las variables de liderazgo son continuas y métricas medidas a través de una escala de 1 a 5 , donde 1 es Nunca y 5 es Frecuentemente, sino es que siempre; según el caso. Los resultados de la prueba también se tienen para los 7 factores: influencia idealizada $(0,270)$, motivación inspirada $(0,472)$, estimulación intelectual $(0,527)$, consideración Individualizada $(0,638)$, recompensa contingente $(0,576)$, gestión por excepción $(0,464)$; el séptimo factor se corresponde con el Liderazgo Laissez-faire. Algunos factores no muestran ser fiables dado que obtienen un valor muy bajo en el estadístico de alfa de Cronbach. Una covarianza baja entre los elementos que componen el factor indica que no existe una relación confiable entre los ítems.

\section{Resultados}

En el Cuadro 1, se presenta el estilo de liderazgo de los directivos universitarios en Colombia, donde se muestra que el estilo que predomina hoy en los directivos es el liderazgo transformacional.

Cuadro 1

Estilos de liderazgo universitarios en Colombia

\begin{tabular}{|l|c|}
\hline Estilo de Liderazgo & Media \\
\hline Transformacional & 12,53105 \\
\hline Transaccional & 12,0867 \\
\hline Laissez Faire & 9,4081 \\
\hline \multicolumn{2}{|c|}{ Fuente: elaboración propia } \\
\hline
\end{tabular}

Al evaluar si existen diferencias significativas entre los estilos de liderazgo transformacional y transaccional de los directivos en Colombia se realiza el test de diferencias de medias asumiendo varianzas diferentes, con la hipótesis nula de igualdad de medias y la hipótesis alternativa diferencia de medias, al obtener un valor $p$ de 0,0013 con un nivel de confianza de $95 \%$ se puede decir que existen diferencias significativas entre la media del liderazgo transformacional y el transaccional y podemos plantear que los directivos colombianos ejercen mayormente un liderazgo transformacional. 
A partir del modelo de análisis planteado por Bass y Avolio (1992) donde se plantea que la puntuación del cuestionario de liderazgo multifactorial se logra sumando tres elementos especificados para cada subfactor. Por ejemplo, el subfactor 1 de influencia idealizada se obtiene sumando las respuestas de los ítems 1,8 y 15 y este procedimiento se sigue para cada uno de los 7 subfactores, se calculan los descriptivos de los estilos de liderazgo según sus subcategorías y se presentan en el Cuadro 2. También se plantea que el rango de puntuación es alto entre los valores $11-15$, es Medio entre $6-10$ y es bajo entre $0-5$.

\section{Cuadro 2}

Estilos de Liderazgo en los Directivos según las subcategorías

\begin{tabular}{|l|l|l|}
\hline & Subcategorías & Media \\
\hline \multirow{4}{*}{ Transformacional } & Influencia Idealizada & 12,0515 \\
\hline & Motivación inspiradora & 12,6581 \\
\hline & Estimulación intelectual & 12,2528 \\
\hline & Consideración Individualizada & 13,1618 \\
\hline \multirow{2}{*}{ Transaccional } & Recompensa contingente & 11,8192 \\
\hline Laissez Faire & Gestión por excepción & 12,3542 \\
\hline & Laissez faire & 9,4081 \\
\hline
\end{tabular}

De lo anterior se plantea que los factores se encuentran ubicados en un nivel Alto a excepción del Laissez Faire que se encuentra en un nivel medio. La Consideración Individualizada $(13,1618)$ es la característica más puntuada por los directivos de las universidades, lo que indicaría que los directivos muestra interés en el bienestar de los demás, asigna proyectos individualmente y presta atención a aquellos que parecen menos involucrados en el grupo. Le siguen la Motivación Inspiradora (12,6581), que muestra que los lideres dan una visión articulada, usan símbolos e imágenes apropiados para ayudar a que los trabajadores generen un significado por su labor, estas dos subcategorías están relacionadas a los procesos de liderazgo transformacional. En el liderazgo transaccional el factor más representativo es la Gestión por excepción (12,3542). El factor que menos puntuación obtiene es el Laissez Faire $(9,4081)$.

En el Cuadro 3, se presenta las medias en los estilos de liderazgo con base en el género del directivo.

\section{Cuadro 3}

Estilo de liderazgo según el género

\begin{tabular}{|c|c|c|c|}
\hline Estilo de Liderazgo & Media Mujeres & Media Hombres & $\begin{array}{l}\text { P-valor asociado al } \\
\text { test de diferencia de } \\
\text { medias }\end{array}$ \\
\hline Transformacional & 12,5248 & 12,2854 & 0,9945 \\
\hline Transaccional & 12,072 & 12,0968 & 0,9098 \\
\hline Laissez Faire & 9,0535 & 9,6562 & 0,0837 \\
\hline
\end{tabular}

Fuente: elaboración propia

Los resultados señalan que las mujeres directivas manifiestan un mayor liderazgo transformacional que los hombres. En el Cuadro 4, se presenta la diferencia de los estilos de liderazgo con el género del directivo universitario en los 7 factores en que se subcategorizan los estilos de liderazgo. 
Cuadro 4

Subcategorías de los Estilos de liderazgo

según el género del directivo universitario

\begin{tabular}{|c|c|c|c|c|}
\hline & \multirow{2}{*}{ Subcategorías } & \multicolumn{2}{|c|}{ Promedio } & \multirow{2}{*}{$\begin{array}{c}\text { P-valor del test de } \\
\text { diferencia de } \\
\text { medias }\end{array}$} \\
\hline & & Mujer & Hombre & \\
\hline \multirow{4}{*}{ Transformacional } & Influencia idealizada & 12,1785 & 11,9625 & 0,3831 \\
\hline & Motivación inspiradora & 12,6517 & 12,6625 & 0,9600 \\
\hline & Estimulación intelectual & 12,1351 & 12,3354 & 0,3818 \\
\hline & $\begin{array}{l}\text { Consideración } \\
\text { individualizada }\end{array}$ & 13,1339 & 12,1812 & 0,8096 \\
\hline \multirow[t]{2}{*}{ Transaccional } & $\begin{array}{l}\text { Recompensa } \\
\text { contingente }\end{array}$ & 11,5765 & 11,9875 & 0,1424 \\
\hline & Gestión por excepción & 12,5675 & 12,2062 & 0,1265 \\
\hline Laissez-faire & Laissez-faire & 9,0535 & 9,6562 & 0,0837 \\
\hline
\end{tabular}

Fuente: elaboración propia

Se evidencia que las universidades dirigidas por mujeres presentan puntajes más altos en Consideración Individualizada $(13,1339)$, Motivación Inspiradora $(12,6517)$ y Gestión por excepción $(12,5675)$, mientras que, en el caso de los hombres tienen mayores puntajes en Motivación Inspiradora $(12,6625)$, Estimulación intelectual $(12,3354)$ y Gestión por excepción $(12,2062)$. Sin embargo, al realizar la prueba estadística para evidenciar que las diferencias son significativas entre hombres y mujeres los resultados muestran que los valores de $\mathrm{p}$ son mayores de 0,05, por lo tanto, no podemos decir que hay diferencias entre el estilo de liderazgo de hombres y mujeres, en los directivos colombianos.

En el Cuadro 5, se presenta la diferencia en los estilos de liderazgo con base en tipo de institución de afiliación del directivo; los resultados muestran que se desarrolla mayormente el estilo transformacional en la institución privada.

\section{Cuadro 5}

Estilos de liderazgo según la afiliación institucional del directivo universitario

\begin{tabular}{|c|c|c|c|}
\hline Estilo de Liderazgo & $\begin{array}{l}\text { Media } \\
\text { Privada }\end{array}$ & Media Publica & $\begin{array}{c}\text { P-valor test } \\
\text { de diferencia } \\
\text { de medias }\end{array}$ \\
\hline Transformacional & 12,5628 & 12,492325 & 0,6733 \\
\hline Transaccional & 12,0766 & 12,09345 & 0,9394 \\
\hline Laissez Faire & 9,4723 & 9,324 & 0,6738 \\
\hline
\end{tabular}

Fuente: elaboración propia

En el Cuadro 6, se presenta el contraste según el tipo de institución de la afiliación del directivo universitario, se encuentra que en las universidades privadas al igual que en las públicas el factor mayor valorado es la Consideración individualizada. Sin embargo, este factor tiene mayor puntaje en las universidades privadas. 


\section{Cuadro 6}

Subcategorías de los Estilos de liderazgo según la afiliación institucional del directivo universitario

\begin{tabular}{|c|c|c|c|c|}
\hline & \multirow[t]{2}{*}{ Subcategorías } & \multicolumn{2}{|c|}{ Promedio } & \multirow{2}{*}{$\begin{array}{l}\text { Prueba t para } \\
\text { diferencia de } \\
\text { medias }\end{array}$} \\
\hline & & Privada & Pública & \\
\hline \multirow{4}{*}{ Transformacional } & Influencia idealizada & 12,0674 & 12,0277 & 0,8739 \\
\hline & $\begin{array}{l}\text { Motivación } \\
\text { inspiradora }\end{array}$ & 12,7177 & 12,5833 & 0,5316 \\
\hline & $\begin{array}{l}\text { Estimulación } \\
\text { intelectual }\end{array}$ & 12,2576 & 12,2380 & 0,9327 \\
\hline & $\begin{array}{l}\text { Consideración } \\
\text { individualizada }\end{array}$ & 13,2085 & 13,1203 & 0,6537 \\
\hline \multirow[t]{2}{*}{ Transaccional } & $\begin{array}{l}\text { Recompensa } \\
\text { contingente }\end{array}$ & 11,8404 & 11,7757 & 0,8190 \\
\hline & Gestión por excepción & 12,3128 & 12,4112 & 0,6808 \\
\hline Laissez Faire & Laissez-faire & 9,4723 & 9,3240 & 0,6738 \\
\hline
\end{tabular}

Fuente: elaboración propia

Al realizar el test de diferencia de medias asumiendo varianzas iguales, teniendo en cuenta los resultados nos muestran que los valores de $p$ están por encima de 0,05 , no podemos decir que hay diferencias significativas entre los estilos de liderazgo de los directivos de instituciones privadas y de las instituciones públicas.

\section{Discusión}

Estos resultados muestran que el factor que más sobresale en los procesos del liderazgo trasnformacional es la consideración individualizada, como lo plantean Avolio y Bass(1995) y Cuadrado (2003) es donde el líder les brinda apoyo, guía y formación de manera individual a sus seguidores, elemento importante en los procesos de transformación donde se reconoce las capacidades y aportes de cada uno dentro de la institución. Seguido de la Motivación inspiradora, donde el líder inspira y motiva a los seguidores a través de desafíos alcanzables, dándoles significado a las metas tanto en lo personal como en lo organizacional. Los resultados del Cuadro 4, son acordes a los que plantea la literatura en estudios de liderazgo y género como el de Khan, Aslam y Riaz (2012) que plantean que las mujeres se carcaterizan por tener un estilo de liderazgo transformacional.

Los resultados descriptivos arrojaron que el estilo de liderazgo con mayor valoración por parte de los Directivos Universitarios fue el estilo Transformacional $(M=12,53)$, en donde se destaca la variable de Consideración Individualizada $(M=13,16)$. Esta característica sugiere que el líder ayuda a sus seguidores a que se autodesarrollen, mostrando interés por ellos, a través de la retroalimentación y atención personalizada. Así mismo, bajo esta característica se utiliza la recompensa para promover la creatividad y el compromiso. Estos resultados estan en linea con Gómez (2020) que planeta que hoy se esperan estilos de liderazgo transformacional que aporten a los cambios y desarrollo de las universidades.

En el caso del analisis de género de los directivos universitarios los resultados presentan que las mujeres directivas desarrollan un estilo transformacional, con valores mas altos en Consideración individualizada $(M=13,13)$ estos resultados están en líneas con los obtenidos por Eagly y Johannesen-Schmidt (2001) que plantean que características como la consideración individualizada y la estimulación intelectual son factores de liderazgo transformacional que se asocian a las mujeres por su orientación a las personas y las relaciones. Estos resultados contribuyen a los estudios de género que muestran cómo éste juega un papel importante en la 
percepción que se tiene sobre el liderazgo ejercido por hombres y mujeres en cargos directivos. Con relación al análisis de tipo de institución a la que pertenece el directivo universitario los resultados presentan que el estilo trasnformacional se desarrolla mas en las instituciones de caracter privado.

Tal como lo plantean Avolio y Bass (1995) y Cuadrado (2003), desde el punto de vista analítico, se evidencia en los hallazgos que los directivos universitarios presentan una orientación al liderazgo transformacional, descartándose aspectos como las consideraciones individualizadas y la motivación inspiradora como factores que ejercen un peso importante dentro de este tipo de liderazgo transformacional. Mientras que en el liderazgo transaccional se destaca la gestión por excepción como factor determinante de este tipo de liderazgo. En el caso del liderazgo Laissez-Faire, este tiene menor presencia en el ejercicio y orientación del liderazgo de los directivos universitarios en Colombia.

Cuando incorporamos la variable género en el análisis de los estilos de liderazgo, encontramos aspectos interesantes de análisis, por ejemplo que las mujeres tienen una orientación mayor hacia la búsqueda de una consideración individualizada, prefieren una interacción que posibilite una motivación inspiradora y puede llegar a trabajar en la búsqueda de desarrollar una gestión por excepción. Mientras que en el caso de los hombres existe una orientación hacia una motivación inspiradora, propicia una estimulación intelectual y se orienta por la gestión por excepción. Lo anterior esta relacionado con lo propuesto por Eagly y Johannesen-Schmidt (2001).

\section{Conclusiones}

El presente estudio buscó describir el estilo de liderazgo de los directivos universitarios en Colombia. Se encontró en los directivos universitarios una orientación hacia el ejercicio del liderazgo transformacional $(12,53105)$. El estilo transformacional de liderazgo está muy orientado por la consideración individualizada y el reconocimiento de las capacidades y apoyo para los procesos de cambio, utilizando símbolos e imágenes que ayudan a los colaboradores a tener una visión más integradora y completa de la organización. También se orienta a los individuos para avanzar hacia grandes desafíos. El otro estilo que se encontró en la investigación con un factor importante fue el liderazgo transaccional $(12,0867)$, fundamentado en la gestión por excepción y la recompensa contingente. Es importante señalar que estadísticamente existen diferencias significativas que demuestran la orientación al estilo transformacional de los directivos universitarios.

De otra parte, al incorporar el componente de genero se evidencia un mayor factor del liderazgo transformacional por parte de las mujeres $(12,5248)$ que por parte de los hombres $(12,2854)$, asi mismo que los hombres $(12,0968)$ presentan una orientación más fuerte que las mujeres $(12,072)$, hacia el liderazgo transaccional y que dentro del liderazgo Laisser- Faire existe una mayor orientación de los hombres $(9,6562)$ hacia este estilo de liderazgo que las mujeres $(9,0535)$ directivas universitarias. Asi mismo, se encontró que al incorporar la naturaleza de la institución los directivos de las instituciones privadas $(12,5628)$ tienen una orientación al liderazgo transformacional mayor que los directivos de las universidades públicas $(12,4923)$. No se encontraron estadísticamente diferencias significativas en los estilos de liderazgo por género, ni diferencias significativas en los estilos de liderazgo cuando se incorpora la afiliación del directivo a una institución bien sea pública y privada.

\section{Referencias bibliográficas}

Altbach, P.G. (2015). Knowledge and education as internacional commodities. Industry and Higher Education, 28, 2-5.

Bass, B.M., y Avolio, B.J. (1992). Organizational Description Questionnaire. Redwood City: Mind Garden. 
Beltrán, W. M. (2013). Del monopolio católico a la explosión pentecostal. Pluralización religiosa, secularización y cambio social en Colombia. Bogotá: Universidad Nacional de Colombia, Facultad de Ciencias Humanas, Centro de Estudios Sociales (CES).

Barbosa, M., Ney Matos, F., Mendonça, J., Paiva, K., y Cassundé, F. (2017). The role of manager: Perceptions from academic-managers of a Brazilian Federal University. Education policy analysis archives, 25, 12. https://doi.org/10.14507/epaa.25.2388.

Black, S. A. (2015). Qualities of Effective Leadership in Higher Education. Open Journal of Leadership, 4, 54-66. http://doi.org/10.4236/ojl.2015.42006.

Brunner, J. J. (2011). Gobernanza universitaria: tipología, dinámicas y tendencias. Revista de Educación, 355, 137-159. http://doi.org/10.1088/1751-8113/44/8/085201.

Castro, D., y Tomàs, M. (2011). Development of Manager-Academics at Institutions of Higher Education in Catalonia. Higher Education Quarterly, 65(3), 290-307. http://doi.org/10.1111/j.1468-2273.2011.00490.x.

Contreras, F. y Barbosa D. (2013). Del liderazgo transaccional al liderazgo transformacional: implicaciones para el cambio organizacional. Revista Virtual Católica del Norte, 39, 152-164.

Creswell, J. W., y Plano, V. L. (2007). Designing and conducting Mixed Methods research. Thousand Oaks, USA: Sage. doi: https://doi.org/10.1177/1558689807306132.

Cuadrado, M. I. (2003). ¿Emplean hombres y mujeres diferentes estilos de liderazgo? Análisis de la influencia de los estilos de liderazgo en el acceso a los puestos de dirección. Revista de Psicología Social, 18(3), 283307. doi: https://doi.org/10.1174/021347403322470864.

Deem, R. (2004). The Knowledge Worker, the Manager-academic and the Contemporary UK University: New and Old Forms of Public Management?. Financial Accountability and Management, 20(May), 107-128. http://doi.org/10.1111/j.1468-0408.2004.00189.x.

Eagly, A. H., y Johannesen-Schmidt, M. C. (2001). The leadership styles of women and men. Journal of Social Issues, 57(4), 781-797. https://doi.org/10.1111/0022-4537.00241.

Esquivias, J. A. (2014). Acerca del ethos profesional del directivo universitario un enfoque antropológico para dirigir la Universidad. Pamplona: Ediciones Universidad de Navarra.

Ferrer, J., Colmenares, F., y Clemenza, C. ( 2010). An Ethical Leader for Change: Strategic Management Platform in Higher Education Institutions. Revista de Ciencias Sociales, 16 (4), 642-653.

Gómez, S. (2020). Estado del arte del liderazgo transformacional en la educación universitaria. Revista Científica Conecta Libertad, 4(1), 75-81.

González, O., González, O., Ríos, G., y León, J.(2013).Características del liderazgo transformacional presentes en un grupo de docentes universitarios. Telos Revista de Estudios Interdisciplinarios en Ciencias Sociales, 15 (3), 355-371.

Hirtz, P, Murray, S.L, y Riordan, C.A. (2007). The effects of leadership on quality. Engineering Management Journal, 19(1), 22-27. https://doi.org/10.1080/10429247.2007.11431718

Khalifa, B. y Ayoubi, R.M. (2015). Leadership styles at Syrian higher education. International Journal of Educational Management, 29(4), 477 - 491. http://dx.doi.org/10.1108/IJEM-03-2014-0036. 
Khan, M. J., Aslam, N., y Riaz, M. N. (2012). Leadership Styles as Predictors of Innovative Work Behavior. Pakistan. Journal of Social and Clinical Psychology, 9(2), 17-22.

Lopera, C. M. (2004). Antinomias, Dilemas y falsas premisas que condicionan la gestión universitaria. Investigación Temática, 9(22), 617-635.

Mendoza, I. A., Ortiz , M.F., y Parker, H. C. (2007). Dos décadas de investigación y desarrollo en liderazgo transformacional. Revista del Centro de Investigación Universidad La Salle, 7(27),25-41.

Mercer, J. (2009). Junior academic-manager in higher education: an untold story?. International Journal of Educational Management, 23(4), 348-359. http://doi.org/10.1108/09513540910957444

Middlehurst, R. (2004). Changing Internal Governance: A Discussion of Leadership Roles and Management Structures in UK Universities. Higher Education Quarterly, 58, 258-279. http://dx.doi.org/10.1111/j.14682273.2004.00273.

Moncayo-Orjuela, B. C., y Zuluaga-Goyeneche, D. (2015). Estilos de liderazgo en cargos universitarios, estudio descriptivo con mujeres Directivas. Panorama, 9(17), 74-84.

Oplatka, I. (2006). Women in educational administration within developing countries: Towards a new international research agenda. Journal of Educational Administration, 44(6), 604-624.

Pérez, G., Jiménez, G., y Romo, G. (2017) Caracterización del liderazgo transformacional de los directivos de instituciones de educación superior. Caso de estudio en una universidad del departamento de Antioquia (Colombia). Entramado, 13 (1), 48-61. http://dx.doi.org/10.18041/entramado.2017v13n1.25137.

Rodríguez-Ponce, E. (2009). El rol de las universidades en la sociedad del conocimiento y en la era de la globalización: evidencia desde Chile. Interciencia, 34 (11), 822 - 829.

Seale, O., y Cross, M. (2015). Leading and managing in complexity: the case of South African deans. Studies in Higher Education, 41(8),1-19. http://do.org/10.1080/03075079.2014.988705.

Soto, A. (2005). Aproximación histórica a la Universidad Colombiana. Revista Historia de la Educación Latinoamericana, 7, 99-136.

Restrepo, J., Trujillo, M., y Guzmán, A. (2012). Gobierno corporativo en las instituciones de educación superior en Colombia. Bogotá: CESA ed.

Winter, R. (2009). Academic manager or managed academic? Academic identity schisms in higher education. Journal of Higher Education Policy and Management, 31(2), 121-131.

http://doi.org/10.1080/13600800902825835. 


\section{Anexo 1. Cuestionario de encuesta}

\begin{tabular}{|c|c|c|c|c|c|}
\hline Pregunta & Nunca & $\begin{array}{l}\text { De vez en } \\
\text { cuando }\end{array}$ & $\begin{array}{l}\text { Algunas } \\
\text { veces }\end{array}$ & $\begin{array}{l}\text { Bastante } \\
\text { a menudo }\end{array}$ & $\begin{array}{l}\text { Frecuente- } \\
\text { mente, sino } \\
\text { es siempre }\end{array}$ \\
\hline \multicolumn{6}{|l|}{ Hago que otros se sientan bien para que estén cerca de mi } \\
\hline \multicolumn{6}{|l|}{ Expreso con pocas palabras lo que podríamos y deberíamos hacer } \\
\hline \multicolumn{6}{|l|}{$\begin{array}{l}\text { Permito que otros piensen sobre problemas del pasado desde nuevas } \\
\text { perspectivas }\end{array}$} \\
\hline \multicolumn{6}{|l|}{ Ayudo a otros a desarrollarse a sí mismo } \\
\hline \multicolumn{6}{|l|}{ Le digo a otros qué hacer si ellos quieren ser premiados por su trabajo } \\
\hline \multicolumn{6}{|l|}{ Estoy satisfecho cuando otros cumplen con los estándares acordados } \\
\hline \multicolumn{6}{|l|}{$\begin{array}{l}\text { Estoy contento permitiéndole a otros continuar con su trabajando de la } \\
\text { misma manera de siempre }\end{array}$} \\
\hline \multicolumn{6}{|l|}{ Proporciono imágenes atrayentes sobre lo que podemos hacer } \\
\hline \multicolumn{6}{|l|}{$\begin{array}{l}\text { Suministro a otros nuevas formas de ver las cosas que son } \\
\text { desconcertantes }\end{array}$} \\
\hline \multicolumn{6}{|l|}{ Permito a otros conocer cómo están haciendo las cosas } \\
\hline \multicolumn{6}{|l|}{$\begin{array}{l}\text { Proporciono reconocimiento/recompensas cuando otros alcanzan sus } \\
\text { objetivos }\end{array}$} \\
\hline \multicolumn{6}{|l|}{ Mientras todo esté marchando, trato de no cambiar las cosas } \\
\hline \multicolumn{6}{|l|}{ Cualquier cosa que quieran los demás, está bien para mí } \\
\hline \multicolumn{6}{|l|}{ Me llama la atención lo que otros pueden obtener por lo que realizan } \\
\hline \multicolumn{6}{|l|}{$\begin{array}{l}\text { Le digo a otros los estándares que deben saber para llevar a cabo su } \\
\text { trabajo }\end{array}$} \\
\hline Le pido a otros lo absolutamente esencial & & & & & \\
\hline
\end{tabular}

Fuente: Elaboracion propia

Esta obra está bajo una Licencia Creative Commons

Attribución-NoCommercial 4.0 International

\section{(cc) BY-NC}

